\title{
BROWNIAN MOTION OF ROTATION
}

BY

\author{
C. D. GORMAN( $(1)$
}

1. Brownian motion of rotation has been studied by F. Perrin [3] and K. Yosida [4]. However both studies are indirect and make use of the partial differential equation of diffusion. In this paper, by a direct application of the formulas for the composition of probabilities, we show how Brownian motion of rotation of the sphere may be constructed from the plane Brownian motion.

Let $(x(t), y(t)), 0 \leqq t<\infty$, be a sample path of the plane Brownian motion process. For each non-negative integer $n$ let the interval $[0,1]$ of real numbers be subdivided by the points $k / 2^{n}, k=0, \cdots, 2^{n}$. Let the polygonal path $\left\{(x, y) \mid x=x^{(n)}(t), y=y^{(n)}(t), 0 \leqq t \leqq 1\right\}$ be defined by:

$$
\begin{aligned}
& x^{(n)}(t)=x\left(\frac{k-1}{2^{n}}\right)+2^{n}\left(t-\frac{k-1}{2^{n}}\right)\left[x\left(\frac{k}{2^{n}}\right)-x\left(\frac{k-1}{2^{n}}\right)\right], \\
& y^{(n)}(t)=y\left(\frac{k-1}{2^{n}}\right)+2^{n}\left(t-\frac{k-1}{2^{n}}\right)\left[y\left(\frac{k}{2^{n}}\right)-y\left(\frac{k-1}{2^{n}}\right)\right],
\end{aligned}
$$

for $(k-1) / 2^{n} \leqq t<k / 2^{n}, k=1, \cdots, 2^{n}, n=0,1, \cdots$.

Let $S$ be a sphere of radius 1 on which has been chosen a point $P$ and an oriented great circle through $P$. Let $S$ be placed on the $(x, y)$-plane such that $P$ coincides with the origin of coordinates and the great circle on $S$ at $P$ is tangent to the positive $x$-axis and is directed in the same sense. Assume now that $S$ rolls without slipping along the polygonal path,

$$
\left\{(x, y) \mid x=x^{(n)}(t), y=y^{(n)}(t), 0 \leqq t \leqq 1\right\}
$$

in a way such that it has the same constant angular velocity along each of the linear portions of the path corresponding to the intervals of time $(k-1) / 2^{n}$ $\leqq t<k / 2^{n}, k=1, \cdots, 2^{n}$. Let $R^{(n)}(t), 0 \leqq t \leqq 1$, be the rotation of $S$ around its center as it rolls in the above manner from the point $(0,0)$ to the point $\left(x^{(n)}(t), y^{(n)}(t)\right)$. In this way, for each sample path of the plane Brownian motion process, a sequence $R^{(n)}(t), 0 \leqq t \leqq 1, n=0,1, \cdots$, of sample paths in the group of rotations in 3-space is obtained.

The main theorem states that $\lim _{n \rightarrow \infty} R^{(n)}(t)$ exists uniformly for $t$ in

Presented to the Society, January 20, 1959; received by the editors December 17, 1958.

(1) This paper is part of a dissertation presented to the faculty of the Graduate School of Yale University in Candidacy for the degree of Doctor of Philosophy. This research was partially supported by the United States Air Force, through the Air Force Office of Scientific Research of the Air Research and Development Command under Contract No. AF 18(600)1127. 
the interval $[0,1]$, for almost all sample paths of the plane Brownian motion process.

This paper is divided into sections which are numbered consecutively from 1 to 5 . The first few sections contain preliminaries. $\$ 4$ establishes the notation and contains a precise statement of the main theorem. In $\$ 5$ the main theorem is proved.

I want to thank my teacher, Professor Shizuo Kakutani for his helpthe kind of help which standard words of acknowledgment can never quite cover.

2. Let $(\Omega, \varepsilon, \operatorname{Pr})$ be a probability space, i.e., $\Omega=\{\omega\}$ is a set of elements, $\mathcal{E}=\{E\}$ is a Borel field of subsets of called "events," and $\operatorname{Pr}$ is a countably additive measure defined on $\mathcal{E}$ satisfying $\operatorname{Pr}\{\Omega\}=1 . \operatorname{Pr}\{E\}$ is called the "probability" of the event $E$. If $x=x(\omega)$ is a random variable, i.e., $x(\omega)$ is a real (complex) valued function defined on $\Omega$ such that $\{\omega \mid x(\omega) \in B\} \in \mathcal{E}$ for every Borel subset $B$ of real (complex) numbers, then $E\{x\}$ shall be the "mean" or "expectation" of $x$, i.e.,

$$
E\{x\}=\int_{\Omega} x(\omega) \operatorname{Pr}(d \omega)
$$

provided the integral exists.

A (mathematical) one-dimensional Brownian motion is a real-valued function $x(t, \omega)$ of the two variables $t$ and $\omega$, defined for all non-negative real numbers $t, 0 \leqq t<\infty$, and for all $\omega \in \Omega$, which has the following properties:

$\left(\mathrm{B}_{1}\right) x(0, \omega) \equiv 0$,

$\left(\mathrm{B}_{2}\right)$ for any real numbers $s, t$ with $0 \leqq s<t<\infty$, the increment $x(t, \omega)$ $-x(s, \omega)$ is $\varepsilon$-measurable in $\omega$ and has a normal distribution with mean 0 and variance $t-s$, i.e.,

$$
E(x, s, t, \alpha)=\{\omega \mid x(t, \omega)-x(s, \omega)<\alpha\} \in \mathcal{E}
$$

and

$$
\operatorname{Pr}\{E(x, s, t, \alpha)\}=\left(1 /(2 \pi(t-s))^{1 / 2}\right) \int_{-\infty}^{\alpha} e^{-u^{2} / 2(t-s)} d u,
$$

for any real number $\alpha$.

$\left(\mathrm{B}_{3}\right)$ for any real numbers $s_{i}, t_{i},(i=1, \cdots, m)$ with $0 \leqq s_{1}<t_{1} \leqq \cdots$ $s_{m}<t_{m}<\infty$, the increments $x\left(t_{i}, \omega\right)-x\left(s_{i}, \omega\right),(i=1, \cdots, m)$, are independent in the sense of probability theory, i.e.,

$$
\operatorname{Pr}\left\{\bigcap_{i=1}^{m} E\left(x, s_{i}, t_{i}, \alpha_{i}\right)\right\}=\prod_{i=1}^{m} \operatorname{Pr}\left\{E\left(x, s_{i}, t_{i}, \alpha_{i}\right)\right\}
$$

for any real numbers $\alpha_{i}(i=1, \cdots, m)$.

A two-dimensional or plane Brownian motion is an ordered pair of two 
mutually independent one-dimensional Brownian motions, i.e., a pair of onedimensional Brownian motions $x(t, \omega)$ and $y(t, \omega)$ with the property that

$$
\begin{aligned}
& \operatorname{Pr}\left\{E(x, s, t, \alpha) \cap E\left(y, s^{\prime}, t^{\prime}, \alpha^{\prime}\right)\right\} \\
& \quad=\operatorname{Pr}\{E(x, s, t, \alpha)\} \cdot \operatorname{Pr}\left\{E\left(y, s^{\prime}, t^{\prime}, \alpha^{\prime}\right)\right\}
\end{aligned}
$$

for any real numbers $s, t, \alpha, s^{\prime}, t^{\prime}, \alpha^{\prime}$ with $0 \leqq s<t<\infty, 0 \leqq s^{\prime}<t^{\prime}<\infty$.

If we consider $B(t, \omega)=(x(t, \omega), y(t, \omega))$ as a point in a Euclidean plane then for each fixed $\omega, B(t, \omega)$ may be considered as a function of $t$ for $0 \leqq t<\infty$, which assumes as values points or vectors in the plane. For fixed $\omega$ the function $B(t, \omega)$ of $t, 0 \leqq t<\infty$, is called a sample path.

The following two lemmas from probability theory shall be needed in the last section of the paper.

LEMMA 1. If $x_{1}, x_{2}, \cdots$ is a sequence of non-negative real-valued random variables and if there exists a sequence $a_{1}, a_{2}, \cdots$ of non-negative constants such that

$$
\begin{aligned}
& \sum_{n=1}^{\infty} a_{n}<\infty, \\
& \sum_{n=1}^{\infty} \operatorname{Pr}\left\{x_{n}>a_{n}\right\}<\infty,
\end{aligned}
$$

then $\sum_{n=1}^{\infty} x_{n}<\infty$, with probability 1 .

The proof of Lemma 1 may be derived from the lemmas of Borel and Cantelli.

Lemma 2. Let $x(t, \omega)$ be one-dimensional Brownian motion. Let the interval $[0,1]$ be subdivided by the points $k / 2^{n}, k=0,1, \cdots, 2^{n}, n=0,1, \cdots$. Let $x_{k}^{(n)}=x_{k}^{(n)}(\omega)=x\left(k / 2^{n}, \omega\right)-x\left((k-1) / 2^{n}, \omega\right)$. Then

$$
\sum_{n=0}^{\infty} \max _{1 \leqq k \leqq 2^{n}}\left|x_{k}^{(n)}(\omega)\right|<\infty
$$

for an w-set having probability 1.

The proof of Lemma 2 may be derived using Lemma 1 together with wellknown properties of Gaussian variables.

3. We consider all rotations of 3-dimensional space around a fixed point (the origin of coordinates). Let $g_{1}$ and $g_{2}$ be two rotations. Then their product $g$ is the rotation obtained by first carrying out the rotation $g_{2}$ and then the rotation $g_{1}$. This is written in the following way: $g=g_{1} \cdot g_{2}$. The set of all rotations form a group which shall be denoted by $G$.

Let a fixed rectangular coordinate system be chosen in 3-space. Then points or vectors $x$ may be represented as triples of real numbers: $\left(x_{1}, x_{2}, x_{3}\right)$. 
Let $|x|$ be the Euclidean length of the vector $x$, i.e., $|x|=\left(x_{1}^{2}+x_{2}^{2}+x_{3}^{2}\right)^{1 / 2}$. Three by three matrices $A$ may be considered as linear transformations on this space of vectors. The notation $|A|$ shall be used to denote the operator norm of $A$, i.e., $|A|=\max _{|x|=1}|A(x)|$.

A rotation $g$ of $G$ may be represented analytically as a 3 by 3 orthogonal matrix $V$ with determinant 1 . The group $G$ shall be topologized by defining the distance between two rotations $g_{1}, g_{2}$ to be $\left|V_{1}-V_{2}\right|$, where $V_{1}, V_{2}$ are the orthogonal matrices corresponding to $g_{1}, g_{2}$ respectively.

4. Let $\Delta$ denote the interval $0 \leqq a \leqq t<b<\infty$ of non-negative real numbers $t$. The notation $x(\Delta, \omega)=x(b, \omega)-x(a, \omega)$ shall be used. Similarly $y(\Delta, \omega)$ $=y(b, \omega)-y(a, \omega)$ and $B(\Delta, \omega)=B(b, \omega)-B(a, \omega)=(x(\Delta, \omega), y(\Delta, \omega))$. Let $\theta(\Delta, \omega)$ be the length of the vector $B(\Delta, \omega)$, i.e., $\theta(\Delta, \omega)=|B(\Delta, \omega)|$, where $|B|=|(x, y)|=\left(x^{2}+y^{2}\right)^{1 / 2}$. Let $\phi(\Delta, \omega)$ denote oriented angle the vector $B(\Delta, \omega)$ makes with the positive $y$-axis. $\phi(\Delta, \omega)$ shall be measured so that the values it assumes lie between $-\pi$ and $\pi$.

For $\phi_{1}, \theta, \phi_{2}$ arbitrary real numbers, define the 3 by 3 real orthogonal matrix $V\left(\phi_{1}, \theta, \phi_{2}\right)$ by

$$
\begin{aligned}
V\left(\phi_{1}, \theta, \phi_{2}\right) & =\left(\begin{array}{ccc}
\cos \phi_{1} & -\sin \phi_{1} & 0 \\
\sin \phi_{1} & \cos \phi_{1} & 0 \\
0 & 0 & 1
\end{array}\right)\left(\begin{array}{ccc}
1 & 0 & 0 \\
0 & \cos \theta & -\sin \theta \\
0 & \sin \theta & \cos \theta
\end{array}\right) \\
& \times\left(\begin{array}{ccc}
\cos \phi_{2} & -\sin \phi_{2} & 0 \\
\sin \phi_{2} & \cos \phi_{2} & 0 \\
0 & 0 & 1
\end{array}\right) .
\end{aligned}
$$

If $\phi, \theta$ are arbitrary real numbers let

$$
V(\phi, \theta)=V(\phi, \theta,-\phi) .
$$

Let the interval $[0,1]$ of real numbers $t, 0 \leqq t \leqq 1$, be subdivided by the points $k / 2^{n}, k=0,1, \cdots, 2^{n}$ for each non-negative integer $n=0,1, \cdots$. Let the subinterval $(k-1) / 2^{n} \leqq t<k / 2^{n}, k=1, \cdots, 2^{n}, n=0,1, \cdots$ be denoted by $\Delta_{k}^{(n)}$. For an arbitrary $t \in[0,1],\left[2^{n} t\right]$ shall denote the largest integer $k$ which is not greater than $2^{n}$. Let $V_{k}^{(n)}=V_{k}^{(n)}(\omega)$ denote the orthogonal matrix $V\left(\phi\left(\Delta_{\mathbf{k}}^{(n)}, \omega\right), \theta\left(\Delta_{\mathbf{k}}^{(n)}, \omega\right)\right), k=1, \cdots, 2^{n}, n=0,1, \cdots$. Let $t$ be an arbitrary real number from $[0,1]$, then $t \in \Delta_{k}^{(n)}$ where $k=\left[2^{n} t\right]+1$. The orthogonal matrix $V\left(\phi\left(\Delta_{k}^{(n)}, \omega\right), 2^{n}\left(t-(k-1) / 2^{n}\right) \theta\left(\Delta_{k}^{(n)}, \omega\right)\right)$ shall be denoted by $V_{i}^{(n)}$ $=V_{t}^{(n)}(\omega), n=0,1, \cdots$. Often, when it seems that there is small probability of confusion, the probability variable $\omega$ shall be omitted from expressions such as $x(t, \omega), B(t, \omega), \theta(\Delta, \omega)$, etc. and these quantities shall be written more simply as $x(t), B(t), \theta(\Delta)$, etc.

Let $t$ be an arbitrary real number from $[0,1]$. Let 


$$
\begin{aligned}
P^{(n)}(t)=P^{(n)}(t, \omega) & =V_{t}^{(n)}(\omega) \cdot V_{\left[2^{n} t\right]}^{(n)}(\omega) \cdots V_{1}^{(n)}(\omega) \\
& =V_{t}^{(n)}(\omega) \cdot \prod_{k=1}^{\left[2^{n} t\right]} V_{k}^{(n)}(\omega),
\end{aligned}
$$

$n=0,1, \cdots$. It shall be convenient to introduce further notation. The rotation of 3-space whose matrix is $V_{k}^{(n)}(\omega)$ shall be denoted by $g_{k}^{(n)}(\omega), k=1, \cdots$, $2^{n}, n=0,1, \ldots$. The rotation of 3 -space whose matrix is $V_{t}^{(n)}(\omega)$ shail be denoted by $g_{t}^{(n)}(\omega), t \in[0,1], n=0,1, \cdots$. Let

$$
\begin{aligned}
g^{(n)}(t) & =g^{(n)}(t, \omega)=g_{t}^{(n)} \cdot g_{\left[2^{n} t\right]}^{(n)} \cdots g_{1}^{(n)} \\
& =g_{t}^{(n)}(\omega) \prod_{k=1}^{\left[2^{n} t\right]} g_{k}^{(n)}(\omega) .
\end{aligned}
$$

THEOREM. The limit,

$$
g(t, \omega)=\lim _{n \rightarrow \infty} g^{(n)}(t, \omega)
$$

exists uniformly in $t, 0 \leqq t \leqq 1$, for $\omega(\in \Omega)$ belonging to an event which has probability 1.

We remark that it follows from the main theorem that $g(t, \omega)$ is a continuous stochastic process in the rotation group $G$. Furthermore, it can be shown that the characteristic matrices [2] $E\left\{T^{l}(g(t))\right\}$ of this process are given by

$$
E\left\{T^{l}(g(t))\right\}=\operatorname{diag}\left\{\cdots e^{\left(l+l^{2}-p^{2}\right) / 2 \cdot t} \cdots\right\}
$$

$p=-l,-l+1, \cdots, l . l=0,1 / 2,1,3 / 2, \cdots$. Here [cf. 1] $T^{l}(g)=\left(T_{p q}^{\prime}(g)\right)$, $l=0,1 / 2,1,3 / 2, \cdots$ is a complete system of mutually inequivalent continuous irreducible representations of $G$ by unitary matrices.

5. In this section a proof of the main theorem shall be given. If $A=\left(a_{i j}\right)$ is a 3 by 3 matrix of real numbers, then $\operatorname{tr}(A)$ denotes the trace of $A$, i.e., $\operatorname{tr}(A)=\sum_{i} a_{i i} . A^{*}$ denotes the transposed matrix of $A$, i.e., the matrix obtained from $A$ by interchanging its rows and columns. Further let

$$
\|A\|=\left(\operatorname{tr}\left(A \cdot A^{*}\right)\right)^{1 / 2}=\left(\sum_{i, j} a_{i j}^{2}\right)^{1 / 2} .
$$

If $|A|$ is the norm of $A$ when it is considered as an operator on the 3-dimensional Euclidean vector space, then

$$
|A| \leqq\|A\| \text {. }
$$

Let $V(\phi, \theta)$ be the real orthogonal matrix defined by (1), then 


$$
V(\phi, \theta)=I+A(\phi, \theta)
$$

for arbitrary $\phi, \theta$, where $I=$ identity matrix and

(5) $A(\phi, \theta)=\left(\begin{array}{llr}-(1-\cos \theta) \sin ^{2} \phi & (1-\cos \theta) \sin \phi \cos \phi & \sin \theta \sin \phi \\ (1-\cos \theta) \sin \phi \cos \phi & -(1-\cos \theta) \cos ^{2} \phi & -\sin \theta \cos \phi \\ -\sin \theta \sin \phi & \sin \theta \cos \phi & -(1-\cos \theta)\end{array}\right)$.

Let the functions $C=C(\theta)$ and $S=S(\theta)$ be defined by the equations

$$
\begin{aligned}
1-\cos \theta & =\frac{\theta^{2}}{2}-\theta^{4} \cdot C(\theta), \\
\sin \theta & =\theta-\theta^{3} \cdot S(\theta) .
\end{aligned}
$$

Then $C$ and $S$ are bounded functions of $\theta,-\infty<\theta<\infty$.

The proof of the following lemma may be demonstrated using (5).

LEMMA 3. Let $\phi$ and $\theta$ be arbitrary real numbers, then

$$
\|A(\phi, \theta)\| \leqq|\theta| \cdot K(|\theta|),
$$

where $K$ is a polynomial which has real non-negative coefficients.

Let the change of variables: $(\phi, \theta) \rightarrow(x, y)$ be defined by

$$
\begin{aligned}
& x=\theta \sin \phi, \\
& y=\theta \cos \phi .
\end{aligned}
$$

Under this change of variables the matrix $A(\phi, \theta)$ is transformed into a matrix which shall be denoted by $A(x, y)$. Using (6), (7) and (8) one may write

$$
A(x, y)=A_{1}(x, y)+A_{3}(x, y)
$$

where

$$
A_{1}(x, y)=\left(\begin{array}{ccc}
-x^{2} / 2 & x y / 2 & x \\
x y / 2 & -y^{2} / 2 & -y \\
-x & y & -\left(x^{2}+y^{2}\right) / 2
\end{array}\right)
$$

and

$$
A_{3}(x, y)=\left(\begin{array}{rrr}
\left(x^{2}+y^{2}\right) x^{2} \cdot C & -\left(x^{2}+y^{2}\right) x y \cdot C & -\left(x^{2}+y^{2}\right) x S \\
-\left(x^{2}+y^{2}\right) x y \cdot C & \left(x^{2}+y^{2}\right) y^{2} \cdot C & \left(x^{2}+y^{2}\right) y \cdot S \\
\left(x^{2}+y^{2}\right) x \cdot S & -\left(x^{2}+y^{2}\right) \cdot y \cdot S & \left(x^{2}+y^{2}\right)^{2} \cdot C
\end{array}\right)
$$

where

$$
C=C\left(\left(x^{2}+y^{2}\right)^{1 / 2}\right), \quad S=S\left(\left(x^{2}+y^{2}\right)^{1 / 2}\right), \quad-\infty<x, y<\infty .
$$


It shall be convenient to introduce the following notational convention: $B_{3}=B_{3}\left(x, y, x^{\prime}, y^{\prime}, \ldots ; C, S, C^{\prime}, S^{\prime}, \ldots\right)$ denotes a 3 by 3 matrix whose elements are polynomials in the variables $x, y, x^{\prime}, y^{\prime}, \cdots, C, S, C^{\prime}, S^{\prime}, \cdots$ any term of which is at least of third degree in the variables $x, y, x^{\prime}, y^{\prime}, \ldots$. Here $C=C\left(\left(x^{2}+y^{2}\right)^{1 / 2}\right), S=S\left(\left(x^{2}+y^{2}\right)^{1 / 2}\right), \quad C^{\prime}=C\left(\left(x^{\prime 2}+y^{\prime 2}\right)^{1 / 2}\right), S^{\prime}$ $=S\left(\left(x^{\prime 2}+y^{\prime 2}\right)^{1 / 2}\right), \cdots,-\infty<x, y, x^{\prime}, y^{\prime}, \cdots<\infty$.

Let $V(x, y)$ denote the matrix into which $V(\phi, \theta)$ is transformed by the change of variables (8).

LeMma 4. Let $(x, y),\left(x^{\prime}, y^{\prime}\right)$ be pairs of real numbers, then

$$
\begin{aligned}
I-V^{*}\left(x+x^{\prime}, y+y^{\prime}\right) \cdot & V(x, y) \cdot V\left(x^{\prime} y^{\prime}\right) \\
& =W\left(x, y, x^{\prime}, y^{\prime}\right)+B_{3}\left(x, y, x^{\prime}, y^{\prime} ; C, S, C^{\prime}, S^{\prime}\right),
\end{aligned}
$$

where

$$
W\left(x, y, x^{\prime}, y^{\prime}\right)=\left(\begin{array}{ccc}
0 & \left(y x^{\prime}-x y^{\prime}\right) / 2 & 0 \\
\left(x y^{\prime}-y x^{\prime}\right) / 2 & 0 & 0 \\
0 & 0 & 0
\end{array}\right) .
$$

Proof. The left-hand side of (12) may be written

$$
\begin{aligned}
I-[I & \left.+A^{*}\left(x+x^{\prime}, y+y^{\prime}\right)\right][I+A(x, y)]\left[I+A\left(x^{\prime}, y^{\prime}\right)\right] \\
= & -A^{*}\left(x+x^{\prime}, y+y^{\prime}\right)-A(x, y)-A\left(x^{\prime}, y^{\prime}\right) \\
& -A^{*}\left(x+x^{\prime}, y+y^{\prime}\right) A(x, y)-A^{*}\left(x+x^{\prime}, y+y^{\prime}\right) A\left(x^{\prime}, y^{\prime}\right) \\
& -A(x, y) A\left(x^{\prime}, y^{\prime}\right)+B_{3} \\
= & -A_{1}^{*}\left(x+x^{\prime}, y+y^{\prime}\right)-A_{1}(x, y)-A_{1}\left(x^{\prime}, y^{\prime}\right) \\
& -A_{1}^{*}\left(x+x^{\prime}, y+y^{\prime}\right) A_{1}(x, y)-A_{1}^{*}\left(x+x^{\prime}, y+y^{\prime}\right) A_{1}\left(x^{\prime}, y^{\prime}\right) \\
& -A_{1}(x, y) A_{1}\left(x^{\prime}, y^{\prime}\right)+B_{3} . \\
A_{1}^{*}(x, y) \cdot A_{1}\left(x^{\prime}, y^{\prime}\right)= & \left(\begin{array}{ccc}
x x^{\prime} & x y^{\prime} & 0 \\
-y x^{\prime} & -y y^{\prime} & 0 \\
0 & 0 & x x^{\prime}+y y^{\prime}
\end{array}\right)+B_{3} . \\
A_{1}(x, y) \cdot A_{1}\left(x^{\prime}, y^{\prime}\right)= & \left.\begin{array}{ccc}
-x x^{\prime} & -x y^{\prime} & 0 \\
x y^{\prime} & y y^{\prime} & 0 \\
0 & 0 & -x x^{\prime}-y y^{\prime}
\end{array}\right)+B_{3} .
\end{aligned}
$$

The left-hand side of (12) may now be written: 


$$
\begin{aligned}
& \left(\begin{array}{ccc}
\left(x+x^{\prime}\right)^{2} / 2 & -\left(x+x^{\prime}\right)\left(y+y^{\prime}\right) / 2 & \left(x+x^{\prime}\right) \\
-\left(x+x^{\prime}\right)\left(y+y^{\prime}\right) & \left(y+y^{\prime}\right)^{2 / 2} & -\left(y+y^{\prime}\right) \\
-\left(x+x^{\prime}\right) & \left(y+y^{\prime}\right) & \left(x+x^{\prime}\right)^{2} / 2+\left(y+y^{\prime}\right)^{2} / 2
\end{array}\right) \\
& +\left(\begin{array}{ccc}
x^{2} / 2 & -x y / 2 & -x \\
-x y / 2 & y^{2} / 2 & y \\
x & -y & \left(x^{2}+y^{2}\right) / 2
\end{array}\right)+\left(\begin{array}{ccc}
x^{\prime 2} / 2 & -x^{\prime} y^{\prime} / 2 & -x^{\prime} \\
-x^{\prime} y^{\prime} / 2 & y^{\prime 2} / 2 & y^{\prime} \\
x^{\prime} & -y^{\prime} & \left(x^{\prime 2}+y^{\prime 2}\right) / 2
\end{array}\right) \\
& +\left(\begin{array}{ccc}
-\left(x+x^{\prime}\right) x & \left(x+x^{\prime}\right) y & 0 \\
\left(y+y^{\prime}\right) \cdot x & -\left(y+y^{\prime}\right) \cdot y & 0 \\
0 & 0 & -\left(x+x^{\prime}\right) x-\left(y+y^{\prime}\right) \cdot y
\end{array}\right) \\
& +\left(\begin{array}{ccc}
-\left(x+x^{\prime}\right) \cdot x^{\prime} & \left(x+x^{\prime}\right) \cdot y^{\prime} & 0 \\
\left(y+y^{\prime}\right) \cdot x^{\prime} & -\left(y+y^{\prime}\right) \cdot y^{\prime} & 0 \\
0 & 0 & -\left(x+x^{\prime}\right) x^{\prime}-\left(y+y^{\prime}\right) \cdot y^{\prime}
\end{array}\right) \\
& +\left(\begin{array}{ccc}
x x^{\prime} & -x y^{\prime} & 0 \\
-y x^{\prime} & y y^{\prime} & 0 \\
0 & 0 & x x^{\prime}+y y^{\prime}
\end{array}\right)+B_{3} \\
& =\left(\begin{array}{ccc}
0 & \left(y x^{\prime}-x y^{\prime}\right) / 2 & 0 \\
\left(x y^{\prime}-y x^{\prime}\right) / 2 & 0 & 0 \\
0 & 0 & 0
\end{array}\right)+B_{3} \\
& =W\left(x, y, x^{\prime}, y^{\prime}\right)+B_{3} \text {. }
\end{aligned}
$$

This completes the proof of Lemma 4.

Lemma 5. Let

$x=x\left(\Delta_{2 k-1}^{(n+1)}, \omega\right), \quad y=y\left(\Delta_{2 k+1}^{(n+1)}, \omega\right), \quad x^{\prime}=x\left(\Delta_{2 k}^{(n+1)}, \omega\right), \quad y^{\prime}=y\left(\Delta_{2 k+1}^{(n+1)}, \omega\right)$ for some $k, 1 \leqq k \leqq 2^{n}$, and $n=0,1, \cdots$, then

$$
\left(2^{n}\right)^{3 / 2}\left\|E\left\{B_{3}\left(x, y, x^{\prime}, y^{\prime} ; C, S, C^{\prime}, S^{\prime}\right)\right\}\right\|
$$

is $a$ bounded function of $n=0,1, \cdots$.

Proof. Let $B_{3}=\left(B_{i j}\right), i, j=1,2,3$. Then

$$
\begin{aligned}
\left(2^{n}\right)^{3 / 2}\left\|E\left\{B_{3}\right\}\right\| & =\left(2^{n}\right)^{3 / 2}\left(\sum_{i, j=1}^{3}\left(E\left\{B_{i j}\right\}\right)^{2}\right)^{1 / 2} \\
& \leqq\left(2^{n}\right)^{3 / 2} \sum_{i, j=1}^{8}\left|E\left\{B_{i j}\right\}\right| .
\end{aligned}
$$


Thus it is sufficient to show that for $i, j=1,2,3$, there are non-negative constants $M_{i j}$ such that

$$
\left(2^{n}\right)^{3 / 2}\left|E\left\{B_{i j}\right\}\right| \leqq M_{i j} \text { (independently of } n \text { ). }
$$

In order to prove this, recall that $B_{i j}$ is a polynomial in the variables $x, y, x^{\prime}$, $y^{\prime}, C, S, C^{\prime}, S^{\prime}$ where $C=C\left(\left(x^{2}+y^{2}\right)^{1 / 2}\right), S=S\left(\left(x^{2}+y^{2}\right)^{1 / 2}\right), S^{\prime}=S^{\prime}\left(\left(x^{\prime 2}+y^{\prime 2}\right)^{1 / 2}\right)$ : $B_{i j}=\sum_{\alpha, \cdots, \delta^{\prime}} A_{\alpha, \ldots, \delta^{\prime}} x^{\alpha} y^{\beta} x^{\prime \alpha^{\prime}} y^{\prime \beta^{\prime}} C^{\gamma} S^{\delta} C^{\prime \gamma^{\prime} S^{\prime \delta^{\prime}}}$ where $\alpha, \cdots, \delta^{\prime}$ take nonnegative integral values, $A_{\alpha}, \ldots, \delta^{\prime}$ are real numbers and $\alpha+\beta+\alpha^{\prime}+\beta^{\prime} \geqq 3$.

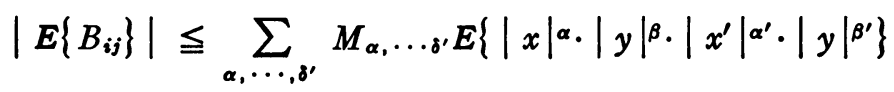

where $M_{\alpha, \cdots, \delta^{\prime}}=\left|A_{\alpha, \ldots, \delta^{\prime}}\right| \cdot \bar{C}^{\gamma+\gamma^{\prime}} \bar{S}^{\delta+\delta^{\prime}}$, here $|C(\theta)| \leqq \bar{C},|S(\theta)| \leqq \bar{S}$ for all $\theta$, $-\infty<\theta<\infty$.

$$
\begin{aligned}
& E\left\{|x|^{\alpha} \cdot|y|^{\beta} \cdot\left|x^{\prime}\right| \alpha^{\prime} \cdot\left|y^{\prime}\right| \beta^{\prime}\right\} \\
&=E\left\{|x|^{\alpha}\right\} \cdot E\left\{|y|^{\beta}\right\} \cdot E\left\{\left|x^{\prime}\right| \alpha^{\prime}\right\} \cdot E\left\{\left|y^{\prime}\right|^{\beta^{\prime}}\right\} \\
& \leqq\left(E\left\{|x|^{2 \alpha}\right\}\right)^{1 / 2}\left(E\left\{|y|^{2 \beta}\right\}\right)^{1 / 2}\left(E\left\{\left|x^{\prime}\right| 2 \alpha^{\prime}\right\}\right)^{1 / 2}\left(E\left\{\left|y^{\prime}\right|^{2 \beta^{\prime}}\right\}\right)^{1 / 2} \\
&=\frac{\nu(\alpha)}{\left(2^{n+1}\right)^{\alpha / 2}} \cdot \frac{\nu(\beta)}{\left(2^{n+1}\right)^{\beta / 2}} \cdot \frac{\nu\left(\alpha^{\prime}\right)}{\left(2^{n+1}\right)^{\alpha^{\prime} / 2}} \cdot \frac{\nu\left(\beta^{\prime}\right)}{\left(2^{n+1}\right)^{\beta^{\prime} / 2}} \cdot
\end{aligned}
$$

Here $\nu(\xi)$ denotes $(2 \xi-1)(2 \xi-3) \cdots 1$. Thus

$$
\begin{aligned}
\left(2^{n}\right)^{3 / 2}\left|E\left\{B_{i j}\right\}\right| & \leqq \sum_{\alpha, \cdots, \delta^{\prime}} M_{\alpha, \cdots, \delta^{\prime}} K_{\alpha, \cdots, \beta^{\prime}}\left(\frac{1}{2^{n}}\right)^{\left(\alpha+\beta+\alpha^{\prime}+\beta^{\prime}-8\right) / 2} \\
& \leqq \sum_{\alpha, \cdots, \delta^{\prime}} M_{\alpha, \cdots, \delta^{\prime}} K_{\alpha, \cdots \beta^{\prime}} \\
& =M_{i j}
\end{aligned}
$$

since $\alpha+\beta+\alpha^{\prime}+\beta^{\prime} \geqq 3$. This completes the proof of Lemma 5 .

Proof of the main theorem. In order to show that

$$
\lim _{n \rightarrow \infty} P^{(n)}(t, \omega)
$$

exists uniformly for $t \in[0,1]$, for an $\omega$-set having probability 1 , it is sufficient to prove that the series

$$
\sum_{n=0}^{\infty} \max _{0 \leqq 1}\left|P^{(n)}(t, \omega)-P^{(n+1)}(t, \omega)\right|
$$

converges with probability 1 .

Let $n=0,1, \cdots$ be fixed, let $t \in[0,1]$ and $k=\left[2^{n} t\right]+1$. Then either $t \in \Delta_{2 k-1}^{(n+1)}$ or $t \in \Delta_{2 k+1}^{(n+1)}$, recalling the definition (2), 


$$
\begin{aligned}
& \left|P^{(n)}(t)-P^{(n+1)}(t)\right| \\
& =\left|V_{t}^{(n)} P^{(n)}\left(\frac{k-1}{2^{n}}\right)-V_{t}^{(n+1)} P^{(n+1)}\left(\frac{2 k-2}{2^{n+1}}\right)\right| \\
& =\mid V_{t}^{(n)} P^{(n)}\left(\frac{k-1}{2^{n}}\right)-V_{t}^{(n+1)} P^{(n)}\left(\frac{k-1}{2^{n}}\right) \\
& +V_{t}^{(n+1)} P^{(n)}\left(\frac{k-1}{2^{n}}\right)-V_{t}^{(n+1)} P^{(n+1)}\left(\frac{2 k-2}{2^{n+1}}\right) \\
& \leqq\left|V_{t}^{(n)}-V_{t}^{(n+1)}\right| \cdot\left|P^{(n)}\left(\frac{k-1}{2^{n}}\right)\right| \\
& +\left|V_{t}^{(n+1)}\right| \cdot\left|P^{(n)}\left(\frac{k-1}{2^{n}}\right)-P^{(n+1)}\left(\frac{2 k-2}{2^{n+1}}\right)\right| .
\end{aligned}
$$

Since $V_{t}^{(n+1)}$ and $P^{(n)}(k-1) / 2^{n}$ are orthogonal

$$
\begin{aligned}
&\left|P^{(n)}(t)-P^{(n+1)}(t)\right| \leqq\left|V_{t}^{(n)}-V_{t}^{(n+1)}\right| \\
&+\left|P^{(n)}\left(\frac{k-1}{2^{n}}\right)-P^{(n+1)}\left(\frac{2 k-2}{2^{n+1}}\right)\right|, \quad t \in \Delta_{2 k-1}^{(n+1)} .
\end{aligned}
$$

Similarly if $t \in \Delta_{2 k}^{(n+1)}$, then

$$
\begin{aligned}
\left|P^{(n)}(t)-P^{(n+1)}(t)\right| \leqq \mid & V_{t}^{(n)}-V_{t}^{(n+1)} V_{2 k-1}^{(n+1)} \mid \\
& \quad+\left|P^{(n)}\left(\frac{k-1}{2^{n}}\right)-P^{(n+1)}\left(\frac{2 k-2}{2^{n+1}}\right)\right| .
\end{aligned}
$$

Using (15) and $\left(15^{\prime}\right)$ one obtains

$$
\begin{aligned}
\max _{0 \leqq t \leqq 1} & \left|P^{(n)}(t)-P^{(n+1)}(t)\right| \\
= & \max _{1 \leqq k \leqq 2^{n}} \cdot \max _{t \in \Delta_{k}^{(n)}}\left|P^{(n)}(t)-P^{(n+1)}(t)\right| \\
\leqq & \max _{1 \leqq k \leqq 2^{n}} \cdot \max _{t \in \Delta_{2 k-1}^{(n+1)}}\left|P^{(n)}(t)-P^{(n+1)}(t)\right| \\
+ & \max _{1 \leqq k \leqq 2^{n}} \cdot \max _{t \in \Delta_{2 k}^{(n+1)}}\left|P^{(n)}(t)-P^{(n+1)}(t)\right| \\
\leqq & 2 \cdot \max _{1 \leqq k \leqq 2^{n}}\left|P^{(n)}\left(\frac{k}{2^{n}}\right)-P^{(n+1)}\left(\frac{2 k}{2^{n+1}}\right)\right| \\
& +\max _{1 \leqq k \leqq 2^{n}} \cdot \max _{t \in \Delta_{2 k-1}^{(n+1)}}\left|V_{t}^{(n)}-V_{t}^{(n+1)}\right| \\
& +\max _{1 \leqq k \leqq 2^{n}} \cdot \max _{t \in \Delta_{2 k}^{(n+1)}}\left|V_{t}^{(n)}-V_{t}^{(n+1)} \cdot V_{2 k-1}^{(n+1)}\right| .
\end{aligned}
$$


Introduce the notation $P_{k}^{(n)}=P^{(n)}\left(k / 2^{n}\right), k=1, \cdots, 2^{n}, n=0,1, \cdots$ In order to prove that (14) converges with probability 1 it is thus sufficient to prove that

$$
\begin{aligned}
& \sum_{n=0}^{\infty} \max _{1 \leqq k \leqq 2^{n}} \cdot \max _{t \in \Delta_{2 k-1}^{(n+1)}}\left|V_{t}^{(n)}(\omega)-V_{t}^{(n+1)}(\omega)\right|, \\
& \sum_{n=0}^{\infty} \max _{1 \leq k \leqq 2^{n}} \cdot \max _{t \in \Delta_{2 k}^{(n+1)}}\left|V_{t}^{(n)}(\omega)-V_{t}^{(n+1)}(\omega) \cdot V_{2 k-1}^{(n+1)}(\omega)\right|
\end{aligned}
$$

and

$$
\sum_{n=0}^{\infty} \max _{1 \leq k \leqq 2^{n}}\left|P_{k}^{(n)}(\omega)-P_{2 k}^{(n+1)}(\omega)\right|
$$

all converge with probability 1 .

The convergence of $(16)$ with probability 1 shall be demonstrated first. Let $t \in \Delta_{2 k-1}^{(n+1)}$, then

$$
V_{t}^{(n)}=V\left(\phi\left(\Delta_{k}^{(n)}\right), \theta_{t}\left(\Delta_{k}^{(n)}\right)\right)=I+A\left(\phi\left(\Delta_{k}^{(n)}\right), \theta_{t}\left(\Delta_{k}^{(n)}\right)\right)
$$

and

$$
\begin{aligned}
V_{t}^{(n+1)} & =V\left(\phi\left(\Delta_{2 k-1}^{(n+1)}\right), \theta_{t}\left(\Delta_{2 k-1}^{(n+1)}\right)\right) \\
& =I+A\left(\phi\left(\Delta_{2 k-1}^{(n+1)}\right), \theta_{t}\left(\Delta_{2 k-1}^{(n+1)}\right)\right) .
\end{aligned}
$$

Here $\theta_{t}\left(\Delta_{k}^{(n)}\right)$ is an abbreviation of $2^{n}\left(t-(k-1) / 2^{n}\right) \theta\left(\Delta_{k}^{(n)}\right)$ and $\theta_{t}\left(\Delta_{2 k-1}^{(n+1)}\right)$ is an abbreviation of $2^{n+1}\left(t-(2 k-2) / 2^{n+1}\right) \theta\left(\Delta_{2 k-1}^{(n+1)}\right)$.

$$
\begin{aligned}
\left|V_{t}^{(n)}-V_{t}^{(n+1)}\right| & \leqq\left|A\left(\phi\left(\Delta_{k}^{(n)}\right), \theta_{t}\left(\Delta_{k}^{(n)}\right)\right)\right|+\left|A\left(\phi\left(\Delta_{2 k-1}^{(n+1)}\right), \theta_{t}\left(\Delta_{2 k-1}^{(n+1)}\right)\right)\right| \\
& \leqq \theta_{t}\left(\Delta_{k}^{(n)}\right) K\left(\theta_{t}\left(\Delta_{k}^{(n)}\right)\right)+\theta_{t}\left(\Delta_{2 k-1}^{(n+1)}\right) K\left(\theta_{t}\left(\Delta_{2 k-1}^{(n+1)}\right)\right),
\end{aligned}
$$

by Lemma 3 . Here $K$ is a polynomial which has real non-negative coefficients. From the above inequality one obtains

$$
\begin{aligned}
& \max _{1 \leqq k \leqq 2^{n}} \max _{t \in \Delta_{2 k-1}^{(n+1)}}\left|V_{t}^{(n)}-V_{t}^{(n+1)}\right| \\
& \leqq \max _{1 \leqq k \leqq 2^{n}}\left[\theta\left(\Delta_{k}^{(n)}\right) K\left(\theta\left(\Delta_{k}^{(n)}\right)\right)+\theta\left(\Delta_{2 k-1}^{(n+1)}\right) K\left(\theta\left(\Delta_{2 k-1}^{(n+1)}\right)\right)\right] \\
& \leqq \max _{1 \leqq k \leqq 2^{n}} \theta\left(\Delta_{k}^{(n)}\right) \cdot K\left(\max _{1 \leqq k \leqq 2^{n}} \theta\left(\Delta_{k}^{(n)}\right)\right) \\
& \quad+\max _{1 \leq k \leq 2^{n}} \theta\left(\Delta_{2 k-1}^{(n+1)}\right) \cdot K\left(\max _{1 \leqq k \leqq 2^{n}} \theta\left(\Delta_{2 k-1}^{(n+1)}\right)\right) .
\end{aligned}
$$


Since $\theta\left(\Delta_{k}^{(n)}\right) \leqq\left|x\left(\Delta_{k}^{(n)}\right)\right|+\left|y\left(\Delta_{k}^{(n)}\right)\right|$, by Lemma 2

$$
\sum_{n=0}^{\infty} \max _{1 \leqq k \leqq 2^{n}} \theta\left(\Delta_{k}^{(n)}\right)<\infty \text { with probability } 1 .
$$

Similarly

$$
\sum_{n=0}^{\infty} \max _{1 \leqq k \leqq 2^{n}} \theta\left(\Delta_{2 k-1}^{(n+1)}\right)<\infty \text {, with probability } 1 .
$$

Let $\max _{1 \leq k \leq 2^{n}} \theta\left(\Delta_{k}^{(n)}\right)=z_{n}, \sum_{0}^{\infty} z_{n}=z<\infty$ with probability 1 . Then $\sum_{0}^{\infty} z_{n} K\left(z_{n}\right)$ $\leqq \sum_{0}^{\infty} z_{n} K(z)=z K(z)$, with probability 1 . It follows that (15) converges with probability 1 . The demonstration that (17) converges with probability 1 is not essentially different from the proof that (15) converges with probability 1. It will be omitted.

Consider the series (18). In order to show that this series converges with probability 1 , use shall be made of Lemma 1 . Thus the probability

$$
\operatorname{Pr}\left\{\max _{1 \leqq k \leqq 2^{n}}\left|P_{k}^{(n)}-P_{2 k}^{(n+1)}\right|>a\right\}
$$

must be estimated. Here $a$ is a positive constant.

Let $Q_{k}^{(n)}=\left\|P_{k}^{(n)}-P_{2 k}^{(n+1)}\right\|^{2}$, then

$$
\begin{aligned}
& \operatorname{Pr}\left\{\max _{1 \leqq k \leqq 2^{n}}\left|P_{k}^{(n)}-P_{2 k}^{(n+1)}\right|>a\right\} \\
& \leqq \operatorname{Pr}\left\{\max _{1 \leqq k \leqq 2^{n}}\left\|P_{k}^{(n)}-P_{2 k}^{(n+1)}\right\|^{2}>a^{2}\right\} \\
& =\operatorname{Pr}\left\{\max _{1 \leqq k \leqq 2^{n}} Q_{k}^{(n)}>a^{2}\right\} .
\end{aligned}
$$

Introduce the $\omega$-sets $E_{k}=E_{k}^{(n)}$ defined by

$$
E_{k}=\left\{Q_{1}^{(n)} \leqq a^{2}, \cdots, Q_{k-1}^{(n)} \leqq a^{2}, Q_{k}^{(n)}>a^{2}\right\},
$$

$k=1, \cdots, 2^{n}, n=0,1, \cdots$. Then

$$
\operatorname{Pr}\left\{\max _{1 \leq k \leq 2^{n}} Q_{k}^{(n)}>a^{2}\right\}=\sum_{k=1}^{2^{n}} \operatorname{Pr}\left\{E_{k}\right\}
$$

Let the real valued random variables $x_{k}=x_{n}^{(n)}(\omega)$ be defined by

$$
x_{k}=\left\{\begin{array}{ll}
1, & \omega \in E_{k} \\
0, & \omega \in \Omega-E_{k},
\end{array} \quad k=1, \cdots, 2^{n} .\right.
$$

Let $\sigma_{k}=x_{1}+\cdots+x_{k}, k=1, \cdots, 2^{n}$. Let $E_{0}=\varnothing, \sigma_{0} \equiv 0$, then 


$$
\begin{aligned}
E\left\{Q_{2^{n}}^{(n)}\right\} & \geqq E\left\{\sigma_{2^{n}} Q_{2^{n}}^{(n)}\right\} \\
& =\sum_{k=1}^{2^{n}} E\left\{\sigma_{k} Q_{k}^{(n)}-\sigma_{k-1} Q_{k-1}^{(n)}\right\} \\
& =\sum_{k=1}^{2^{n}} E\left\{x_{k} Q_{k}^{(n)}+\sigma_{k-1}\left(Q_{k}^{(n)}-Q_{k-1}^{(n)}\right)\right\} \\
& =\sum_{k=1}^{2^{n}} E\left\{x_{k} \cdot Q_{k}^{(n)}\right\}+\sum_{k=1}^{2^{n}} E\left\{\sigma_{k-1}\left(Q_{k}^{(n)}-Q_{k-1}^{(n)}\right)\right\} \\
& \geqq a^{2} \sum_{k=1}^{2^{n}} \operatorname{Pr}\left\{E_{k}\right\}+\sum_{k=1}^{2^{n}} E\left\{\sigma_{k-1}\left(Q_{k}^{(n)}-Q_{k-1}^{(n)}\right)\right\}
\end{aligned}
$$

Combining this inequality with (20) one obtains

$$
\begin{aligned}
\operatorname{Pr}\left\{\max _{1 \leqq k \leqq 2^{n}} Q_{k}^{(n)}>a^{2}\right\} \leqq \frac{1}{a^{2}}\left[E\left\{Q_{2^{n}}^{(n)}\right\}-\sum_{k=1}^{2^{n}} E\left\{\sigma_{k-1}\left(Q_{k}^{(n)}-Q_{k-1}^{(n)}\right)\right\}\right] \\
E\left\{\sigma_{k-1}\left(Q_{k}^{(n)}-Q_{k-1}^{(n)}\right)\right\} \\
=E\left\{\sigma_{k-1}\left(\left\|P_{k}^{(n)}-P_{2 k}^{(n+1)}\right\|^{2}-\left\|P_{k-1}^{(n)}-P_{2 k-2}^{(n+1)}\right\|^{2}\right)\right\} \\
=E\left\{\sigma _ { k - 1 } \operatorname { t r } \left[\left(P_{k}^{(n)}-P_{2 k}^{(n+1)}\right) \cdot\left(P_{k}^{(n) *}-P_{2 k}^{(n+1) *}\right)\right.\right. \\
\left.\left.-\left(P_{k-1}^{(n)}-P_{2 k-2}^{(n+1)}\right) \cdot\left(P_{k-1}^{(n) *}-P_{2 k-2}^{(n+1) *}\right)\right]\right\} \\
=2 E\left\{\sigma_{k-1} \cdot \operatorname{tr}\left[P_{2 k-2}^{(n+1)} P_{k-1}^{(n) *}-P_{2 k}^{(n+1)} P_{k}^{(n) *}\right]\right\}
\end{aligned}
$$

By definition of $P_{k}^{(n)}$ and $P_{2 k}^{(n+1)}$ :

Thus

$$
P_{k}^{(n)}=V_{k}^{(n)} P_{k-1}^{(n)}, \quad P_{2 k}^{(n+1)}=V_{2 k}^{(n+1)} P_{2 k-1}^{(n+1)} P_{2 k-2}^{(n+1)} .
$$

$$
\begin{aligned}
\operatorname{tr} & {\left[P_{2 k-2}^{(n+1)} P_{k-1}^{(n) *}-P_{2 k}^{(n+1)} P_{k}^{(n) *}\right] } \\
& =\operatorname{tr}\left(P_{2 k-2}^{(n+1)} P_{k-1}^{(n) *}\right)-\operatorname{tr}\left(V_{2 k}^{(n+1)} V_{2 k-1}^{(n+1)} P_{2 k-2}^{(n+1)} P_{k-1}^{(n) *} V_{k}^{(n) *}\right) \\
& =\operatorname{tr}\left(P_{2 k-2}^{(n+1)} P_{k-1}^{(n) *}\right)-\operatorname{tr}\left(V_{k}^{(n) *} V_{2 k}^{(n+1)} V_{2 k-1}^{(n+1)} P_{2 k-2}^{(n+1)} P_{k-1}^{(n) *}\right) \\
& =\operatorname{tr}\left[\left(I-V_{k}^{(n) *} V_{2 k}^{(n+1)} V_{2 k-1}^{(n+1)}\right) \cdot\left(P_{2 k-2}^{(n+1)} P_{k-1}^{(n) *}\right)\right] . \\
2 E & \left\{\sigma_{k-1} \operatorname{tr}\left[P_{2 k-2}^{(n+1)} P_{k-1}^{(n) *}-P_{2 k}^{(n+1)} P_{k}^{(n) *}\right]\right\} \\
& =2 \operatorname{tr}\left[E\left\{\left(I-V_{k}^{(n) *} V_{2 k}^{(n+1)} V_{2 k-1}^{(n+1)}\right) \cdot\left(\sigma_{k-1} P_{2 k-2}^{(n+1)} P_{k-1}^{(n) *}\right)\right\}\right] \\
& =2 \operatorname{tr}\left[E\left\{\left(I-V_{k}^{(n) *} V_{2 k}^{(n+1)} V_{2 k-1}^{(n+1)}\right)\right\} \cdot E\left\{\sigma_{k-1} P_{2 k-2}^{(n+1)} P_{k-1}^{(n) *}\right\}\right] .
\end{aligned}
$$

Since $P_{2 k-2}^{(n+1)} P_{k-1}^{(n) *}$ is orthogonal and $\sigma_{k-1}$ may take only the values 0 and 1 , any element of the matrix $E\left\{\sigma_{k-1} P_{2 k-2}^{(n+1)} P_{k-1}^{(n) *}\right\}$ is in absolute value no greater than 1. Thus: 


$$
\begin{aligned}
& \left|E\left\{\sigma_{k-1}\left(Q_{k}^{(n)}-Q_{k-1}^{(n)}\right)\right\}\right| \\
& \quad=\left|2 \cdot \operatorname{tr}\left[E\left\{I-V_{k}^{(n) *} V_{2 k}^{(n+1)} \cdot V_{2 k-1}^{(n+1)}\right\} \cdot E\left\{\sigma_{k-1} P_{2 k-2}^{(n+1)} P_{k-1}^{(n) *}\right\}\right]\right| \\
& \quad \leqq 2 \cdot 3 \cdot\left\|E\left\{I-V_{k}^{(n) *} V_{2 k}^{(n+1)} V_{2 k-1}^{(n+1)}\right\}\right\| .
\end{aligned}
$$

Hence

$$
\left|E\left\{\sigma_{k-1}\left(Q_{k}^{(n)}-Q_{k-1}^{(n)}\right)\right\}\right| \leqq 6 \cdot\left\|E\left\{I-V_{k}^{(n) *} \cdot V_{2 k}^{(n+1)} V_{2 k-1}^{(n+1)}\right\}\right\| .
$$

Similarly one may compute

$$
\left|E\left\{Q_{k}^{(n)}-Q_{k-1}^{(n)}\right\}\right| \leqq 6 \cdot\left\|E\left\{I-V_{k}^{(n) *} \cdot V_{2 k}^{(n+1)} \cdot V_{2 k-1}^{(n+1)}\right\}\right\| .
$$

Turning to the evaluation of

$$
\begin{gathered}
E\left\{I-V_{k}^{(n) *} V_{2 k}^{(n+1)} V_{2 k-1}^{(n+1)}\right\}: \\
V_{k}^{(n) *}=V^{*}\left(\phi\left(\Delta_{k}^{(n)}\right), \theta\left(\Delta_{k}^{(n)}\right)\right)=V^{*}\left(x\left(\Delta_{k}^{(n)}\right), y\left(\Delta_{k}^{(n)}\right)\right), \\
V_{2 k}^{(n+1)}=V\left(\phi\left(\Delta_{2 k}^{(n+1)}\right), \theta\left(\Delta_{2 k}^{(n+1)}\right)\right)=V\left(x\left(\Delta_{2 k}^{(n+1)}\right), y\left(\Delta_{2 k}^{(n+1)}\right)\right), \\
V_{2 k-1}^{(n+1)}=V\left(\phi\left(\Delta_{2 k-1}^{(n+1)}\right), \theta\left(\Delta_{2 k-1}^{(n+1)}\right)\right)=V\left(x\left(\Delta_{2 k-1}^{(n+1)}\right), y\left(\Delta_{2 k-1}^{(n+1)}\right)\right) .
\end{gathered}
$$

Let $x=x\left(\Delta_{2 k}^{(n+1)}\right), \quad y=y\left(\Delta_{2 k}^{(n+1)}\right), \quad x^{\prime}=x\left(\Delta_{2 k-1}^{(n+1)}\right), \quad y^{\prime}=y\left(\Delta_{2 k-1}^{(n+1)}\right)$, then $x+x^{\prime}$ $=x\left(\Delta_{k}^{(n)}\right), y+y^{\prime}=y\left(\Delta_{k}^{(n)}\right)$. By Lemma 4 :

$$
\begin{aligned}
\| E\{I & \left.-V_{k}^{(n) *} V_{2 k}^{(n+1)} V_{2 k-1}^{(n+1)}\right\} \| \\
& =\left\|E\left\{I-V^{*}\left(x+x^{\prime}, y+y^{\prime}\right) V(x, y) V\left(x^{\prime}, y^{\prime}\right)\right\}\right\| \\
& =\left\|E\left\{W\left(x, y, x^{\prime}, y^{\prime}\right)\right\}+E\left\{B_{3}\right\}\right\| \\
& =\left\|E\left\{B_{3}\right\}\right\|,
\end{aligned}
$$

$E\{W\}$ being the zero matrix because the gaussian variables $x, y, x^{\prime}, y^{\prime}$ are independent and have mean zero. Now

$$
\left\|E\left\{B_{3}\right\}\right\|=\left(\frac{1}{2^{n}}\right)^{3 / 2} b_{n}, \quad n=0,1, \cdots,
$$

where by Lemma $5, b_{n}$ is a bounded sequence of non-negative real numbers. Thus

$$
\begin{array}{r}
\left|E\left\{\sigma_{k-1}\left(Q_{k}^{(n)}-Q_{k-1}^{(n)}\right)\right\}\right| \leqq\left(\frac{1}{2^{n}}\right)^{3 / 2} \cdot 6 b_{n}, \\
\left|E\left\{Q_{k}^{(n)}-Q_{k-1}^{(n)}\right\}\right| \leqq\left(\frac{1}{2^{n}}\right)^{8 / 2} \cdot 6 \cdot b_{n},
\end{array}
$$

$k=1, \cdots, 2^{n}, n=0,1, \cdots$. From these inequalities one obtains: 


$$
\begin{aligned}
E\left\{Q_{2^{n}}^{(n)}\right\} & =E\left\{\sum_{k=1}^{2^{n}}\left(Q_{k}^{(n)}-Q_{k-1}^{(n)}\right)\right\} \\
& \leqq \sum_{k=1}^{2^{n}}\left|E\left\{Q_{k}^{(n)}-Q_{k-1}^{(n)}\right\}\right| \leqq\left(\frac{1}{2^{n}}\right)^{1 / 2} \cdot 6 b_{n} .
\end{aligned}
$$

Similarly

$$
\left|\sum_{k=1}^{2^{n}} E\left\{\sigma_{k-1}\left(Q_{k}^{(n)}-Q_{k-1}^{(n)}\right)\right\}\right| \leqq\left(\frac{1}{2^{n}}\right)^{1 / 2} \cdot 6 \cdot b_{n} .
$$

Let the estimates (23) and (24) be applied to (21). One obtains

$$
\operatorname{Pr}\left\{\max _{1 \leqq k \leqq 2^{n}} Q_{k}^{(n)}>a^{2}\right\} \leqq \frac{1}{a^{2}}\left(\frac{1}{2^{n}}\right)^{1 / 2} \cdot\left(12 b_{n}\right) .
$$

Finally

$$
\operatorname{Pr}\left\{\max _{1 \leqq k \leqq 2^{n}}\left|P_{k}^{(n)}-P_{2 k}^{(n+1)}\right|>a\right\} \leqq \frac{1}{a^{2}} \cdot\left(\frac{1}{2^{n}}\right)^{1 / 2} \cdot c_{n}
$$

$n=0,1,2, \ldots$ where $c_{n}$ is a bounded sequence of non-negative real numbers. In (26) let $a=a_{n}=2^{-n / 8}$ then $\sum_{n=0}^{\infty} a_{n}<\infty$ and

$$
\operatorname{Pr}\left\{\max _{1 \leqq k \leqq 2^{n}}\left|P_{k}^{(n)}-P_{2 k}^{(n+1)}\right|>a_{n}\right\} \leqq\left(\frac{1}{2^{n}}\right)^{1 / 4} \cdot c_{n}
$$

so that $\sum_{n=0}^{\infty} \operatorname{Pr}\left\{\max _{1 \leqq k \leqq 2^{n}}\left|P_{k}^{(n)}-P_{2 k}^{(n+1)}\right|>a_{n}\right\}<\infty$. From Lemma 1 , one may now conclude that

$$
\sum_{n=0}^{\infty} \max _{1 \leqq k \leqq 2^{n}}\left|P_{k}^{(n)}(\omega)-P_{2 k}^{(n+1)}(\omega)\right|<\infty,
$$

for an $\omega$-set of probability 1 . This proves that (18) converges with probability 1 and completes the proof of the main Theorem.

\section{REFERENCES}

1. I. M. Gelfand and Z. Ya. Sapiro, Representations of the group of rotations in three-dimensional space and their applications, Amer. Math. Soc. Translations (2) vol. 2 (1956) pp. 207-316.

2. Y. Kawada and K. Itô, On the probability distributions on a compact group I, Proceedings of the Physico-Mathematical Society of Japan ser. 3 vol. 22 (1940) pp. 977-998.

3. F. Perrin, Etude mathématique du mouvement Brownien de rotation, Ann. Sci. Ecole Norm. Sup. ser. 3 vol. 45 (1928) pp. 1-51.

4. K. Yosida, Brownian motion on the surface of the 3-sphere, Ann. Math. Statist. vol. 20 (1949) pp. 292-296.

UNIVERSITY OF WASHINGTON, SEATtLE, WASHington 